\title{
An Adaptive Regulator for Space Teleoperation System in Task Space
}

\author{
Chao Ge, Weiwei Zhang, Hong Wang, and Xiaoyi Li \\ Institute of Information Engineering, Hebei United University, Tangshan, Hebei 063009, China \\ Correspondence should be addressed to Chao Ge; gechao365@126.com
}

Received 26 June 2014; Accepted 20 July 2014; Published 6 August 2014

Academic Editor: Zheng-Guang Wu

Copyright (C) 2014 Chao Ge et al. This is an open access article distributed under the Creative Commons Attribution License, which permits unrestricted use, distribution, and reproduction in any medium, provided the original work is properly cited.

\begin{abstract}
The problem of the gravity information which can not be obtained in advance for bilateral teleoperation is studied. In outer space exploration, the gravity term changes with the position changing of the slave manipulator. So it is necessary to design an adaptive regulator controller to compensate for the unknown gravity signal. Moreover, to get a more accurate position tracking performance, the controller is designed in the task space instead of the joint space. Additionally, the time delay considered in this paper is not only time varying but also unsymmetrical. Finally, simulations are presented to show the effectiveness of the proposed approach.
\end{abstract}

\section{Introduction}

A teleoperation system can extend the human-sensing and manipulative capabilities to the remote environment. Now the teleoperation system has been used in many areas, for instance, outer space operation, robotic telesurgery, handling of toxic, and harmful materials, and underwater exploration [1-3].

In bilateral teleoperation system, the master and the slave manipulators are connected via a long communication network. So the time delay cannot be ignored. It has been noticed that the presence of time delay can influence the system performance. And it can even destabilize a stable system. It is recognized that Anderson and Spong [4] proposed the first delay independent controller for constant time delays via the scattering transformation. Later, the wave variables method was introduced [5]. Then the wave variables method had been improved by many papers [6-8]. However, it is necessary to recognize the fact that the classic scattering transformation may lead to position drift. Then a proportional derivative plus damping $(\mathrm{PD}+\mathrm{d})$ controller was proposed in [9] and the scattering transformation approach was discarded. Later, a simple proportional plus damping $(\mathrm{P}+\mathrm{d})$ controller was proposed in [10]. However, the time delay considered in this paper was constant. In [11], the model-free linear observer-based PD output feedback control design was used to solve the output feedback stabilization for industrial robotic systems. Reference [12] presented a hybrid control strategy for the trajectory tracking control problem of robotic manipulators. The use of Internet for teleoperation communication provides obvious benefits. Nevertheless, the time delay caused by Internet communication channel is not only time varying but also unsymmetrical. Time delay has become an unavoidable factor affecting the stability of closedloop system [13-17].

Thanks to $[18,19]$, a simple $\mathrm{P}+\mathrm{d}$ controller can ensure position tracking and asymptotic convergence to zero of velocities in the presence of variable time delays.

Comparing with other control methods, the PD controller has irreplaceable advantages. Owing to its simple structure and easy implementation, it has been used extensively. However, in [9-12], it is easy to see that an assumption was needed before designing the controller, in which the gravity information can be achieved in advance. However, in many practical applications, it is a serious restriction for the application of PD controller in teleoperation system. Moreover, this issue arises for the teleoperation applications of the robots in the outer space. In outer space, different 
locations have different gravity acceleration. However, with more and more advanced technology, it is possible to detect the gravity. It is expensive and may take a long time and it cannot adapt to the requirements of real-time control. So an adaptive regulator is necessary.

In the existing literatures about the teleoperation system, most controllers were designed in joint space. And the objectives of most papers were to design a controller to make the joint position error between the master and the slave convergence to zero. However, the ultimate aim of using teleoperation is to make the slave complete a certain job under the control of the human operator. And the job is completed by the terminal actuator. It is necessary to notice the phenomenon where the master and the slave manipulators have different joint lengths and joint mass and even different dynamics models may exist. Under this phenomenon, the joint synchronization cannot guarantee the terminal actuator synchronization. This situation can be avoided with the controller designing in the task space [20].

In some literatures for space teleoperation [21-24], the gravity term was directly neglected when the gravity acceleration was zero. In this paper, we consider the situation that gravity information cannot be obtained. An adaptive regulator is added in the typical $\mathrm{P}+\mathrm{d}$ controller to compensate for the unknown gravity signal. Moreover, to make the slave manipulator complete its job accurately under domination of the human operator, the controller is designed in task space. Consider the Internet communication channel; the time delay considered in our paper is time varying and asymptotical.

This paper is organized as follows. Section 2 presents the modeling framework and the related properties and assumptions. The controller is proposed and the stability analysis is deprived in Section 3. In Section 4, some simulation results are applied to prove the effectiveness of the approach. Finally, some conclusion will be provided and some future work will be mentioned.

\section{Problem Formulation}

Consider a master-slave bilateral teleoperation system modeled as a pair of $n$-degree-of-freedom (DOF) serial links with revolute joints. The nonlinear dynamics are presented as

$$
\begin{gathered}
M_{m}\left(q_{m}\right) \ddot{q}_{m}+C_{m}\left(q_{m}, \dot{q}_{m}\right) \dot{q}_{m}+G_{m}\left(q_{m}\right)=\tau_{m}-J_{m}^{T}\left(q_{m}\right) F_{h}, \\
M_{s}\left(q_{s}\right) \ddot{q}_{s}+C_{s}\left(q_{s}, \dot{q}_{s}\right) \dot{q}_{s}+G_{s}\left(q_{s}\right)=J_{s}^{T}\left(q_{s}\right) F_{e}-\tau_{s},
\end{gathered}
$$

where $m$ represents the master manipulator and $s$ represents the slave manipulator. $q_{m}(t), q_{s}(t) \in \mathbb{R}^{n}$ are the vectors of the joint displacements; $\dot{q}_{m}(t), \dot{q}_{s}(t) \in \mathbb{R}^{n}$ are the vectors of joint velocities; $\ddot{q}_{m}(t), \ddot{q}_{s}(t) \in \mathbb{R}^{n}$ are the vectors of joint accelerations; $M_{m}\left(q_{m}\right), M_{s}\left(q_{s}\right): \mathbb{R}^{n} \rightarrow \mathbb{R}^{n \times n}$ are the positive definite inertia matrices; $C_{m}\left(q_{m}, \dot{q}_{m}\right), C_{s}\left(q_{s}, \dot{q}_{s}\right)$ : $\mathbb{R}^{n} \times \mathbb{R}^{n} \rightarrow \mathbb{R}^{n \times n}$ are the matrices of centripetal and coriolis torques; $G_{m}\left(q_{m}\right), G_{s}\left(q_{s}\right): \mathbb{R}^{n} \rightarrow \mathbb{R}^{n}$ are the gravitational torques; $F_{h}, F_{e} \in \mathbb{R}^{n}$ are the human operator force and the environment force, respectively; $J_{m}\left(q_{m}\right), J_{s}\left(q_{s}\right) \in \mathbb{R}^{n \times n}$ are the
Jacobian matrices for the master manipulator and the salve manipulator; $\tau_{m}, \tau_{s} \in \mathbb{R}^{n}$ are the applied torques.

Here the following assumption for Jacobian matrix $J_{i}\left(q_{i}\right)(i=m, s)$ is introduced.

Assumption 1. The Jacobian matrix $J_{i}\left(q_{i}\right)(i=m, s)$ should be nonsingular.

Remark 2. As we all know, in reality industry production, the singularity problem may exist in the Jacobian matrix. Then the $\left\|J_{i}\left(q_{i}\right)\right\| \rightarrow \infty$ and the torque of system will tend to infinity when there exists singularity in Jacobian matrix. Thanks to the new technology, this situation can be avoided by institutional design and trajectory planning. So this assumption in this paper is reasonable.

It is well known that the work space of manipulator can be transformed from joint space to task space with a nonsingular Jacobian matrix. So we have

$$
\begin{gathered}
x_{i}=h_{i}\left(q_{i}\right), \\
\dot{x}_{i}=J_{i}\left(q_{i}\right) \dot{q}_{i}, \\
\ddot{x}_{i}=\dot{J}_{i}\left(q_{i}\right) \dot{q}_{i}+J_{i}\left(q_{i}\right) \ddot{q}_{i},
\end{gathered}
$$

where $x_{i}$ is the joint position in task space and $i=m, s$.

For the sake of simplicity, the arguments of functions are eliminated hereafter. And the master and slave robot dynamics in the task space are presented as follows:

$$
\begin{gathered}
M_{t m} \ddot{x}_{m}+C_{t m} \dot{x}_{m}+G_{t m}=J_{m}^{-T} \tau_{m}-F_{h}, \\
M_{t s} \ddot{x}_{s}+C_{t s} \dot{x}_{s}+G_{t s}=F_{e}-J_{s}^{-T} \tau_{s},
\end{gathered}
$$

where

$$
\begin{aligned}
M_{t m} & =J_{m}^{-T} M_{m} J_{m}^{-1}, \\
M_{t s} & =J_{s}^{-T} M_{s} J_{s}^{-1}, \\
C_{t m} & =J_{m}^{-T}\left(C_{m}-M_{m} J_{m}^{-1} \dot{J}_{m}\right) J_{m}^{-1}, \\
C_{t s} & =J_{s}^{-T}\left(C_{s}-M_{s} J_{s}^{-1} \dot{J}_{s}\right) J_{s}^{-1}, \\
G_{t m} & =J_{m}^{-T} G_{m}, \\
G_{t s} & =J_{s}^{-T} G_{s} .
\end{aligned}
$$

Below, fundamental properties of the master and slave manipulators needed in the subsequent analysis are presented.

Property 1. The inertia matrix $M_{t i}(q)$ is positive definite function, and there exist positive constants $m_{1}$ and $m_{2}$ such that

$$
m_{1} I \leq M_{t i}(q) \leq m_{2} I
$$

Property 2. The matrix $\dot{M}_{t i}(q)-2 C_{t i}(q, \dot{q})$ is skew-symmetric.

Property 3. For all $q_{i}, x, y \in R^{n \times 1}$, there exists a positive scalar $\alpha_{i}$ such that $\left\|C_{i}(q, x) y\right\| \leq \alpha_{i}\|x\|\|y\|$. 
Property 4. The gravity functions $G_{t m}$ and $G_{t s}$ can be linearized and can be expressed as $G_{t m}\left(q_{m}\right)=Y_{m} a_{m}$ and $G_{t s}\left(q_{s}\right)=Y_{s} a_{s}$, where $Y_{m}$ and $Y_{s}$ are known nonlinear functions and usually are called regressor matrices, while $a_{m}$ and $a_{s}$ are unknown vectors.

The following standard assumptions are imposed on the teleoperation system.

Assumption 3. The variable time delay has an upper bound $\bar{T}_{i}$; that is, $0 \leq T_{i}(t) \leq \bar{T}_{i} \leq \infty, i=m, s$.

Assumption 4. Following standard considerations, we assume that the human operator and the environment are passive (force to velocity) maps; that is, there exist scalars $\kappa_{i} \in R_{0}^{+}$such that for all $t \geq 0$

$$
\int_{0}^{t} \dot{x}_{m}^{T} F_{h} d \sigma \geq-\kappa_{m}, \quad-\int_{0}^{t} \dot{x}_{s}^{T} F_{e} d \sigma \geq-\kappa_{s} .
$$

Before proposing the controller, the following lemma is needed.

Lemma 5. For any vector signals $x, y$, any variable time delay $0 \leq T_{i}(t) \leq \bar{T}_{i} \leq \infty$, and constant $\alpha>0$, one has

$$
-2 \int_{0}^{t} x^{T}(\sigma) \int_{-T_{i}(\sigma)}^{0} y(\sigma+\theta) d \theta d \sigma \leq \alpha\|x\|_{2}^{2}+\frac{\bar{T}_{i}^{2}}{\alpha}\|y\|_{2}^{2} .
$$

\section{Controller Design}

In original researches, little attention was paid to the gravity term; moreover, they even made the assumption that the gravity can be accurately obtained in advance. However, in many cases, the gravity not only cannot be accurately obtained directly, but also cannot be measured. This issue arises for the teleoperation applications of the robots in the outer space. It is well known that the gravity coefficient $g$ is different for the earth, outer space, and the moon. The above two issues may render that the precise value of parameter $g$ is not known for teleoperation. In this section, we consider this problem and propose a new controller combining a typical $\mathrm{P}+\mathrm{d}$ controller and an adaptive regulator.

Without the accurate gravity information, the new direct force-feedback controller combined with the estimate gravity information is designed as follows:

$$
\begin{aligned}
\tau_{m} & =J_{m}^{T}\left[K_{m}\left(x_{s}\left(t-T_{s}(t)\right)-x_{m}\right)-B_{m} \dot{x}_{m}+\widehat{G}_{t m}\right], \\
\tau_{s} & =J_{s}^{T}\left[K_{s}\left[x_{s}-x_{m}\left(t-T_{m}(t)\right)\right]+B_{s} \dot{x}_{s}-\widehat{G}_{t s}\right],
\end{aligned}
$$

where $K_{m}, K_{s}, B_{m}$, and $B_{s}$ are positive constant scalars and the $\widehat{G}_{t m}$ and $\widehat{G}_{t s}$ are the estimations for $G_{t m}$ and $G_{t s}$, respectively. In addition, they are described as follows:

$$
\begin{aligned}
\widehat{G}_{t m} & =Y_{m} \widehat{a}_{m}, \\
\widehat{G}_{t s} & =Y_{s} \widehat{a}_{s},
\end{aligned}
$$

where $\widehat{a}_{m}(t)$ and $\widehat{a}_{s}(t)$ are the adaptive matrices with the tuning laws as

$$
\begin{aligned}
& \dot{\widehat{a}}_{m}(t)=-\Gamma_{m} Y_{m} \dot{x}_{m}^{T}, \\
& \dot{a}_{s}(t)=-\Gamma_{s} Y_{s} \dot{x}_{s}^{T},
\end{aligned}
$$

where $\Gamma_{m}$ and $\Gamma_{s}$ are positive definite matrices.

Theorem 6. For the teleoperation system (1) controlled by (8) with Assumptions 1-4, the closed-loop system is stable with the tuning laws (10) and the following condition holds:

$$
2 B_{m} B_{s}>K_{m} K_{s}\left(\bar{T}_{m}^{2}+\bar{T}_{s}^{2}\right)
$$

And the velocity and position errors are bounded (i.e., $\left.\dot{x}_{i}, x_{m}-x_{s} \in \ell_{\infty}\right)$ and $\dot{x}_{i} \in \ell_{2} ;$ moreover $\left|x_{m}-x_{s}\left(t-T_{s}(t)\right)\right| \epsilon$ $\ell_{\infty}$

Proof. Let us consider the Lyapunov function candidates:

$$
\begin{gathered}
V_{1}=\frac{1}{2} \dot{x}_{m}^{T} M_{t m} \dot{x}_{m}+\frac{K_{m}}{2 K_{s}} \dot{x}_{s}^{T} M_{t s} \dot{x}_{s}+\frac{K_{m}}{2}\left|\left(x_{m}-x_{s}\right)\right|^{2} \\
+\int_{0}^{t}\left(\dot{x}_{m}^{T} F_{h}-\frac{K_{m}}{K_{s}} \dot{x}_{s}^{T} F_{e}\right) d \sigma+k_{m}+\frac{K_{m} k_{s}}{K_{s}}, \\
V_{2}=\frac{1}{2} \tilde{a}_{m}^{T} \Gamma_{m}^{-1} \tilde{a}_{m}+\frac{K_{m}}{2 K_{s}} \tilde{a}_{s}^{T} \Gamma_{s}^{-1} \tilde{a}_{s},
\end{gathered}
$$

where $\widetilde{a}_{m}=\widehat{a}_{m}-a_{m}$ and $\tilde{a}_{s}=\widehat{a}_{s}-a_{s}$.

Property 1 and Assumption 4 guarantee that the function $V_{1}$ is positive definite and it is obvious that $V_{2}$ is also positive definite with the positive $\Gamma_{m}$ and $\Gamma_{s}$.

The derivative of the above function $V_{1}$ is presented as follows:

$$
\begin{aligned}
\dot{V}_{1}= & \dot{x}_{m}^{T}\left(-C_{t m} \dot{x}_{m}-G_{t m}+J_{m}^{-T} \tau_{m}-F_{h}\right)+\frac{1}{2} \dot{x}_{m}^{T} \dot{M}_{t m} \dot{x}_{m} \\
& +\frac{K_{m}}{K_{s}} \dot{x}_{s}^{T}\left(-C_{t s} \dot{x}_{s}-G_{t s}+F_{e}-J_{s}^{-T} \tau_{s}\right)+\frac{1}{2} \dot{x}_{s}^{T} \dot{M}_{t s} \dot{x}_{s} \\
& +K_{m}\left(\dot{x}_{m}-\dot{x}_{s}\right)\left(x_{m}-x_{s}\right)+\dot{x}_{m}^{T} F_{h}-\frac{K_{m}}{K_{s}} \dot{x}_{s}^{T} F_{e}
\end{aligned}
$$

with Property 2 and the new controller (11) the subsequent equation can be given as

$$
\begin{aligned}
\dot{V}_{1}= & \dot{x}_{m}^{T}\left[K_{m}\left(x_{s}\left(t-T_{s}(t)\right)-x_{m}\right)-B_{m} \dot{x}_{m}+\widetilde{G}_{t m}\right] \\
& +\frac{K_{m}}{K_{s}} \dot{x}_{s}^{T}\left[K_{s}\left[x_{m}\left(t-T_{m}(t)\right)-x_{s}\right]-B_{s} \dot{x}_{s}+\widetilde{G}_{t s}\right] \\
& +K_{m}\left(\dot{x}_{m}^{T}-\dot{x}_{s}^{T}\right)\left(x_{m}-x_{s}\right) \\
= & -\dot{x}_{m}^{T} B_{m} \dot{x}_{m}-\frac{K_{m}}{K_{s}} \dot{x}_{s}^{T} B_{s} \dot{x}_{s}+\dot{x}_{m}^{T} K_{m}\left(x_{s}\left(t-T_{s}(t)\right)-x_{s}\right) \\
& +\dot{x}_{s}^{T} K_{m}\left(x_{m}\left(t-T_{m}(t)\right)-x_{m}\right)+\dot{x}_{m}^{T} \widetilde{G}_{t m}+\frac{K_{m}}{K_{s}} \dot{x}_{s}^{T} \widetilde{G}_{t s},
\end{aligned}
$$


where $\widetilde{G}_{t m}=\widehat{G}_{t m}-G_{t m}=Y_{m}\left(\widehat{a}_{m}-a_{m}\right)$ and $\widetilde{G}_{t s}=\widehat{G}_{t s}-G_{t s}=$ $Y_{s}\left(\widehat{a}_{s}-a_{s}\right)$. With the following equation:

$$
x_{i}\left(t-T_{i}(t)\right)-x_{i}(t)=-\int_{-T_{i}(t)}^{0} \dot{x}_{i}(t+\theta) d \theta,
$$

we can get

$$
\begin{aligned}
\dot{V}_{1}= & -B_{m}\left|\dot{x}_{m}\right|^{2}-\frac{K_{m}}{K_{s}} B_{s}\left|\dot{x}_{s}\right|^{2}-K_{m} \dot{x}_{m}^{T} \int_{-T_{s}(t)}^{0} \dot{x}_{s}(t+\theta) d \theta \\
& -K_{m} \dot{x}_{s}^{T} \int_{-T_{m}(t)}^{0} \dot{x}_{m}(t+\theta) d \theta+\dot{x}_{m}^{T} \widetilde{G}_{t m}+\frac{K_{m}}{K_{s}} \dot{x}_{s}^{T} \widetilde{G}_{t s} .
\end{aligned}
$$

The derivative of the above function $V_{2}$ is given as follows:

$$
\dot{V}_{2}=\tilde{a}_{m}^{T} \Gamma_{m}^{-1} \dot{\widehat{a}}_{m}+\tilde{a}_{s}^{T} \Gamma_{s}^{-1} \dot{\widehat{a}}_{s}
$$

with the adaptive tuning law (11) yielding

$$
\begin{aligned}
\dot{V}_{2}= & \tilde{a}_{m}^{T} \Gamma_{m}^{-1}\left(-\Gamma_{m} Y_{m} \dot{x}_{m}\right)+\frac{K_{m}}{K_{s}} \tilde{a}_{s}^{T} \Gamma_{s}^{-1}\left(-\Gamma_{s} Y_{s} \dot{x}_{s}\right) \\
= & -\left(Y_{m} \tilde{a}_{m}\right)^{T} \dot{x}_{m}-\frac{K_{m}}{K_{s}}\left(Y_{s} \tilde{a}_{s}\right)^{T} \dot{x}_{s}, \\
\dot{V}= & \dot{V}_{1}+\dot{V}_{2}=-B_{m}\left|\dot{x}_{m}\right|^{2}-\frac{K_{m}}{K_{s}} B_{s}\left|\dot{x}_{s}\right|^{2} \\
& -K_{m} \dot{x}_{m}^{T} \int_{-T_{s}(t)}^{0} \dot{x}_{s}(t+\theta) d \theta \\
& -K_{m} \dot{x}_{s}^{T} \int_{-T_{m}(t)}^{0} \dot{x}_{m}(t+\theta) d \theta .
\end{aligned}
$$

Now let us invoke Lemma 5 to obtain a bound on the integral of $\dot{V}$. Toward this end, integrate the above equation from 0 to $t$, yielding

$$
\begin{aligned}
V(t)-V(0) \leq & -B_{m}\left\|\dot{x}_{m}\right\|^{2}-\frac{K_{m}}{K_{s}} B_{s}\left\|\dot{x}_{s}\right\|^{2} \\
& +\frac{K_{m} \beta_{m}}{2}\left\|\dot{x}_{m}\right\|^{2}+\frac{K_{m} \bar{T}_{s}^{2}}{2 \beta_{m}}\left\|\dot{x}_{s}\right\|^{2} \\
& +\frac{K_{m} \beta_{s}}{2}\left\|\dot{x}_{s}\right\|^{2}+\frac{K_{m} \bar{T}_{m}^{2}}{2 \beta_{s}}\left\|\dot{x}_{m}\right\|^{2} \\
= & -\left(B_{m}-\frac{K_{m} \beta_{m}}{2}-\frac{K_{m} \bar{T}_{m}^{2}}{2 \beta_{s}}\right)\left\|\dot{x}_{m}\right\|^{2} \\
& -\left(\frac{K_{m}}{K_{s}} B_{s}-\frac{K_{m} \beta_{s}}{2}-\frac{K_{m} \bar{T}_{s}^{2}}{2 \beta_{m}}\right)\left\|\dot{x}_{s}\right\|^{2}
\end{aligned}
$$

Thus, satisfying condition (12) with the nonnegativity of $V, \dot{x}_{i} \in \ell_{2}$ can be proved; moreover, $\dot{x}_{i}, x_{m}-x_{s} \in \ell_{\infty}$ and $\left|x_{m}-x_{s}\left(t-T_{s}(t)\right)\right| \in \ell_{\infty}$ can be proved.

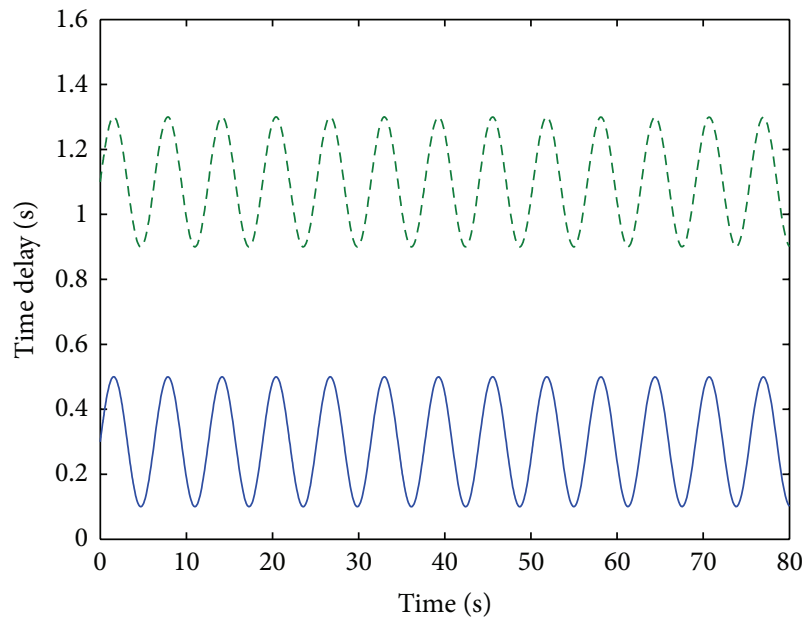

$-T_{m}(t)$

$--T_{s}(t)$

Figure 1: The time delay.

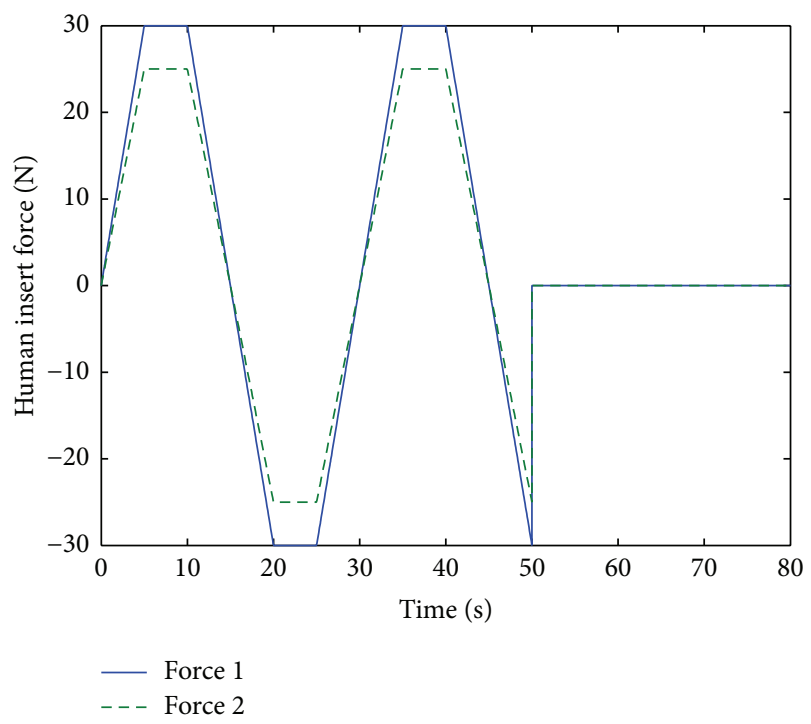

FIGURE 2: The human insert force.

Remark 7. In this paper, we consider the situation that gravity information cannot be obtained. An adaptive regulator is added in the typical $\mathrm{P}+\mathrm{d}$ controller to compensate for the unknown gravity signal.

Remark 8. In order to make the slave manipulator complete its job accurately under domination of the human operator, the controller is designed in task space. Consider the Internet communication channel; the time delay considered in this paper is time varying and asymptotical.

Remark 9. From the above proof, we can see that the adaptive tuning law is derived in the paper. Therefore, we can obtain less conservative result by optimizing these parameters. 


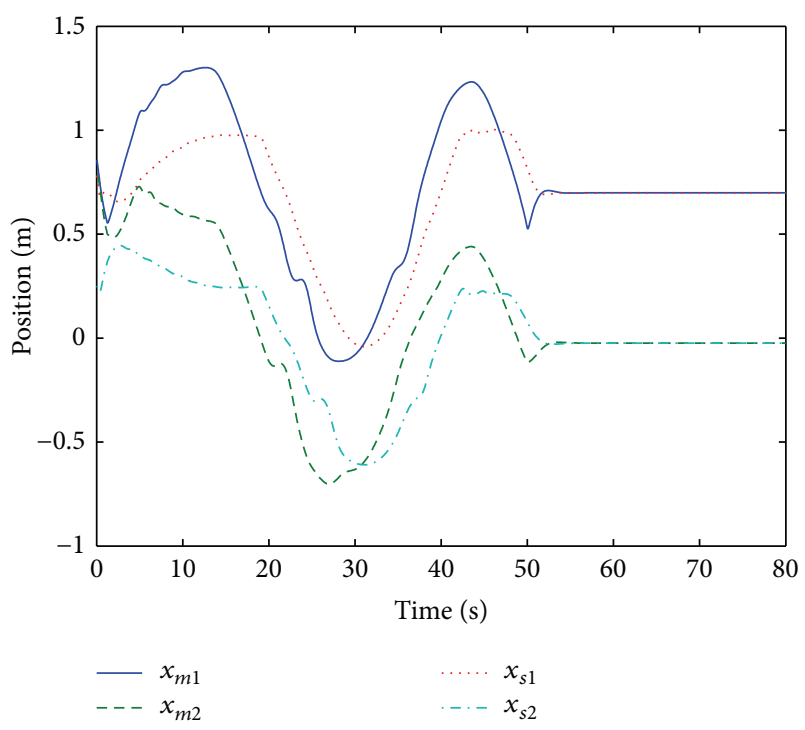

FIGURE 3: The positions of the master and the slave in task space with the known gravity term.

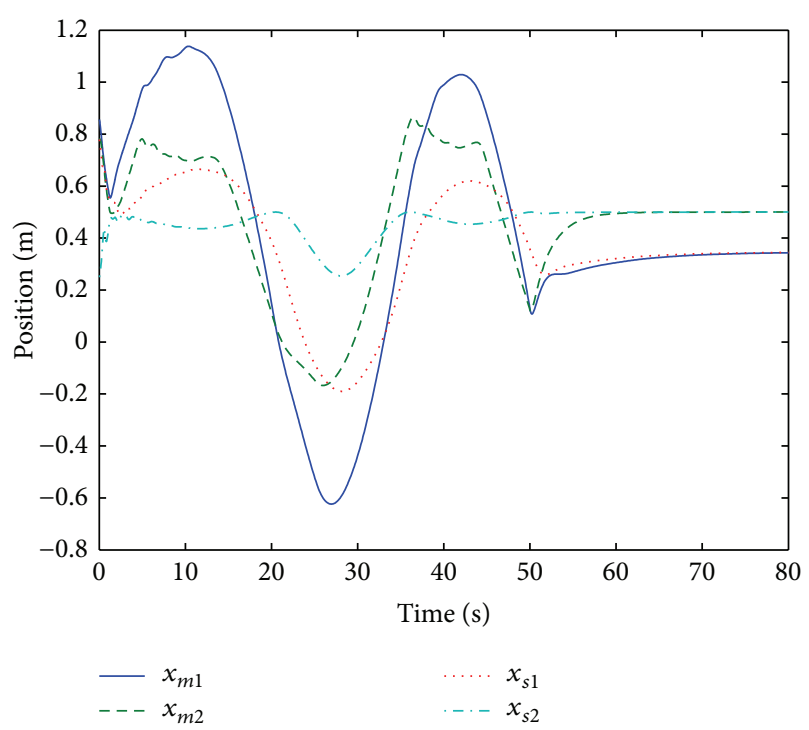

Figure 4: The positions of the master and the slave in task space without adaptive regulator.

The numerical example given in the next part shows the effectiveness of our result.

\section{Simulations}

In order to show the effectiveness of the proposed scheme, in this section some simulations are presented in which the local and remote manipulators are modeled as a pair of $2 \mathrm{DOF}$ serial links.

$$
\begin{gathered}
M_{m}\left(q_{m}\right) \ddot{q}_{m}+C_{m}\left(q_{m}, \dot{q}_{m}\right) \dot{q}_{m}+G_{m}\left(q_{m}\right)=\tau_{m}-J_{m}^{T}\left(q_{m}\right) F_{h}, \\
M_{s}\left(q_{s}\right) \ddot{q}_{s}+C_{s}\left(q_{s}, \dot{q}_{s}\right) \dot{q}_{s}+G_{s}\left(q_{s}\right)=J_{s}^{T}\left(q_{s}\right) F_{e}-\tau_{s},
\end{gathered}
$$

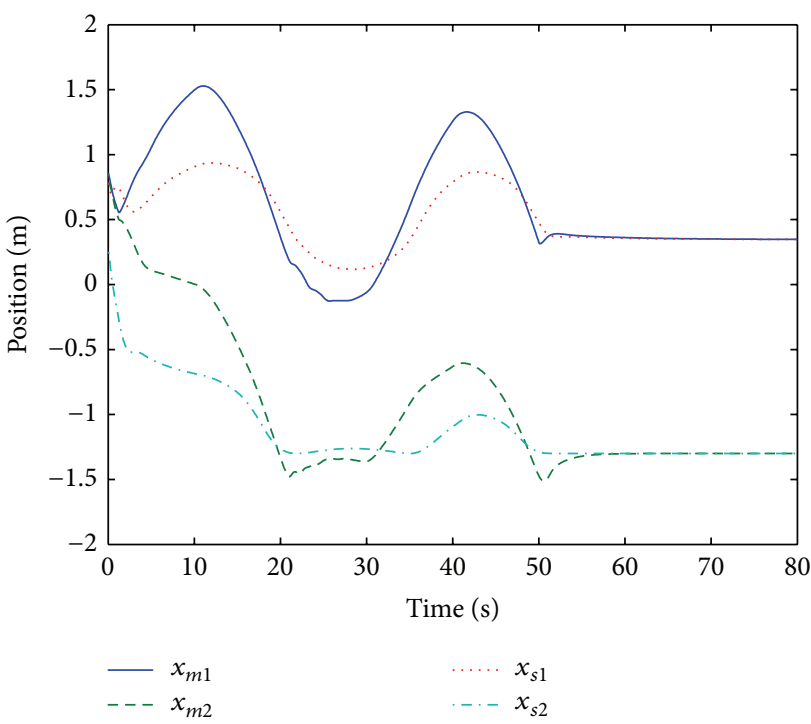

FIGURE 5: The positions of the master and the slave in task space with adaptive regulator.

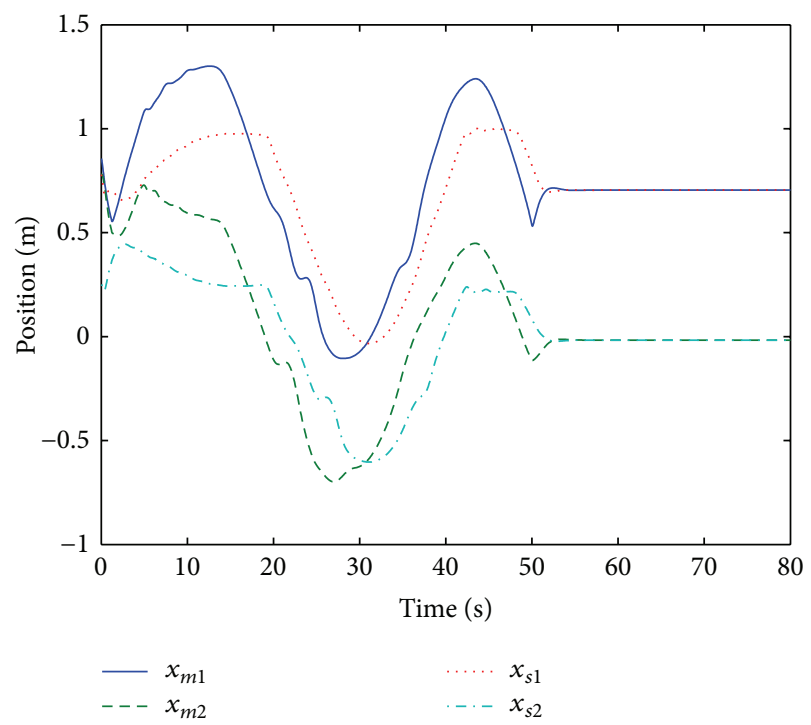

FIGURE 6: The positions of the master and the slave in task space with the known gravity term.

where

$$
\begin{gathered}
M_{m}(q)=M_{s}(q)=\left[\begin{array}{cc}
M_{11} & M_{12} \\
* & M_{22}
\end{array}\right], \\
C_{m}(q, \dot{q})=C_{s}(q, \dot{q})=\left[\begin{array}{ll}
C_{11} & C_{12} \\
C_{21} & C_{22}
\end{array}\right], \\
G_{m}(q)=G_{s}(q)=\left[\begin{array}{l}
G_{1} \\
G_{2}
\end{array}\right]
\end{gathered}
$$

in which

$$
\begin{aligned}
& M_{11}=\left(2 l_{1} \cos \left(q_{2}\right)+l_{2}\right) l_{2} m_{2}+l_{1}^{2}\left(m_{1}+m_{2}\right), \\
& M_{12}=l_{2}^{2} m_{2}+l_{1} l_{2} m_{2} \cos \left(q_{2}\right),
\end{aligned}
$$




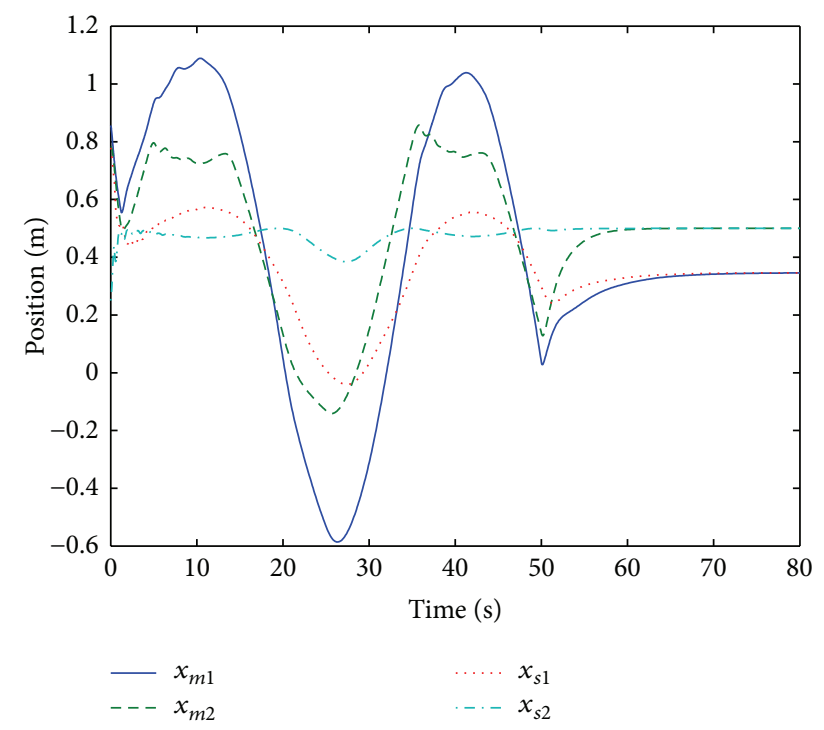

FIGURE 7: The positions of the master and the slave in task space without adaptive regulator.

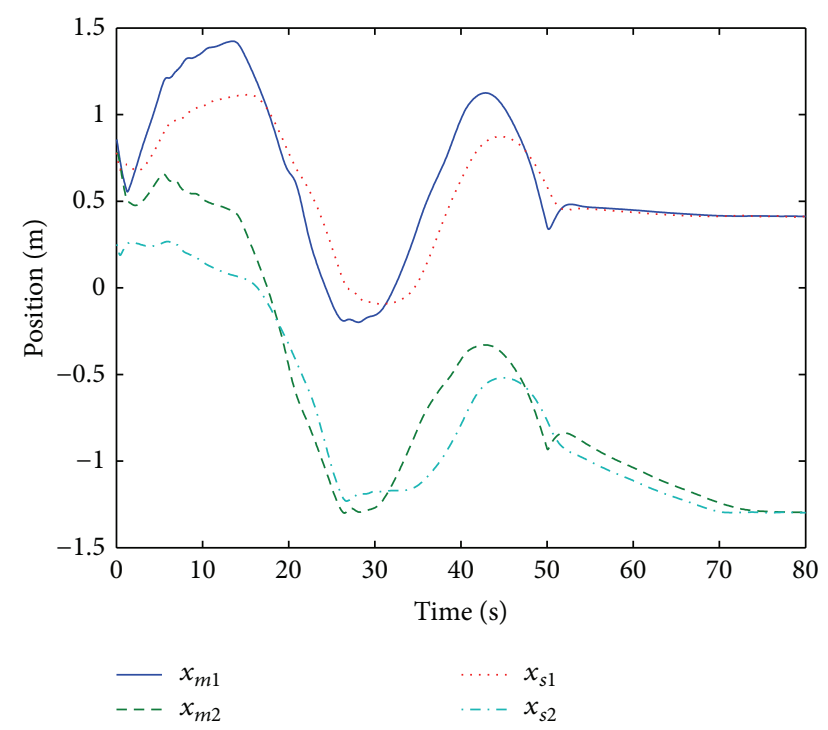

FIGURE 8: The positions of the master and the slave in task space with adaptive regulator.

$$
\begin{aligned}
M_{21} & =M_{12}, \\
M_{22} & =l_{2}^{2} m_{2}, \\
C_{11} & =-l_{1} l_{2} m_{2} \sin \left(q_{2}\right) \dot{q}_{2}, \\
C_{12} & =-l_{1} l_{2} m_{2} \sin \left(q_{2}\right)\left(\dot{q}_{1}+\dot{q}_{2}\right), \\
C_{21} & =l_{1} l_{2} m_{2} \sin \left(q_{2}\right) \dot{q}_{1}, \\
C_{22} & =0, \\
G_{1} & =g\left(m_{2} l_{2} \cos \left(q_{1}+q_{2}\right)+\left(m_{1}+m_{2}\right) l_{1} \cos \left(q_{1}\right)\right), \\
G_{2} & =g m_{2} l_{2} \cos \left(q_{1}+q_{2}\right) .
\end{aligned}
$$

The manipulator Jacobian matrix $J_{i}\left(q_{i}\right)$ mapping from joint space to task space is given as

$$
J_{i}=\left[\begin{array}{ll}
J_{11} & J_{12} \\
J_{21} & J_{22}
\end{array}\right]
$$

in which

$$
\begin{aligned}
& J_{11}=-l_{1} \sin \left(q_{1}\right)-l_{2} \sin \left(q_{1}+q_{2}\right), \\
& J_{12}=-l_{2} \sin \left(q_{1}+q_{2}\right), \\
& J_{21}=l_{1} \cos \left(q_{1}\right)+l_{2} \cos \left(q_{1}+q_{2}\right), \\
& J_{22}=l_{2} \cos \left(q_{1}+q_{2}\right) .
\end{aligned}
$$

Moreover, with the detailed definition of $a_{m}^{T}, a_{s}^{T}, Y_{m}$, and $Y_{s}, G_{t m}$ and $G_{t s}$ can follow this definition:

$$
G_{t}=J^{-T}\left[\begin{array}{cc}
\cos \left(q_{1}+q_{2}\right) & \cos \left(q_{1}\right) \\
\cos \left(q_{1}+q_{2}\right) & 0
\end{array}\right]\left[\begin{array}{c}
g m_{2} l_{2} \\
g l_{1}\left(m_{1}+m_{2}\right)
\end{array}\right] .
$$

For simulation, in the master manipulator we choose the parameters $m_{1}=10 \mathrm{~kg}, m_{2}=7 \mathrm{~kg}, l_{1}=0.7 \mathrm{~m}$, and $l_{2}=0.5 \mathrm{~m}$ and the master manipulator usually placed in the laboratory; therefore, we can set the $g=9.81 \mathrm{~m} / \mathrm{s}^{2}$. The slave manipulator parameters are $m_{1}=7 \mathrm{~kg}, m_{2}=5 \mathrm{~kg}$, $l_{1}=0.5 \mathrm{~m}, l_{2}=0.4 \mathrm{~m}$, and $g=0.2 \mathrm{~m} / \mathrm{s}^{2}$. The controller parameters are set as $K m=20, B_{m}=40, K s=40$, $B s=60$, and $\Gamma_{s}=0.1$. The initial joint configurations of the master and slave are set as $x_{m}(0)=\left[\begin{array}{lll}0.7830 & 0.8562\end{array}\right]^{T}$ and $x_{s}(0)=\left[\begin{array}{lll}0.7794 & 0.25\end{array}\right]^{T}$. The simulation results are used to verify the following: when we move the master robot, does the slave manipulator follow the master manipulator? Based on Theorem 6, the delays are set as $T_{m}(t)=0.3+0.2 \sin (t)$ and $T_{s}(t)=1.1+0.2 \sin (t)$. Figure 1 shows the time delay we set in this paper. The human operation inserting forces are presented in Figure 2. Figure 3 shows the positions of $x_{m}$ and $x_{s}$ when the gravity term can be obtained in advance and $g=5 \mathrm{~m} / \mathrm{s}^{2}$. Figure 4 shows the positions of $x_{m}$ and $x_{s}$ without the adaptive regulator and $g=5 \mathrm{~m} / \mathrm{s}^{2}$. Figure 5 shows the positions of $x_{m}$ and $x_{s}$ with the adaptive regulator and $g=5 \mathrm{~m} / \mathrm{s}^{2}$. Figure 6 shows the positions of $x_{m}$ and $x_{s}$ when the gravity term can be obtained in advance and $g=1 \mathrm{~m} / \mathrm{s}^{2}$. Figure 7 shows the positions of $x_{m}$ and $x_{s}$ without the adaptive regulator and $g=1 \mathrm{~m} / \mathrm{s}^{2}$. Figure 8 shows the positions of $x_{m}$ and $x_{s}$ with the adaptive regulator and $g=1 \mathrm{~m} / \mathrm{s}^{2}$.

From Figure 3, we can see that the slave moves with the master when the gravity term is known in advance. However, in Figure 4, without the adaptive regulator, the slave does not move with the master, specially, in the $x_{s 2}$ direction. In Figure 5, the problem is solved by applying the new controller we proposed in this paper. By comparing Figures 7 and 8, we also can get the same conclusion. 


\section{Conclusion}

In this paper, the case that the gravity term cannot be obtained is considered in outer space exploration. An adaptive regulator is proposed to compensate for the unknown gravity term. By proposing the proper Lyapunov function, the adaptive tuning law is also derived in our paper. Moreover, the simulation results prove the effectiveness of our controller. In this paper, we consider that the Jacobian matrix is accurately known. However, in some cases, the Jacobian matrix cannot be known. Therefore, in the future, we will consider using adaptive theory to estimate the unknown Jacobian matrix.

\section{Conflict of Interests}

The authors declare that there is no conflict of interests regarding the publication of this paper.

\section{References}

[1] C. Mitsantisuk, K. Ohishi, and S. Katsura, "Estimation of action/reaction forces for the bilateral control using Kalman filter," IEEE Transactions on Industrial Electronics, vol. 59, no. 11, pp. 4383-4393, 2012.

[2] I. G. Polushin, X. P. Liu, and C. H. Lung, "Stability of bilateral teleoperators with generalized projection-based force reflection algorithms," Automatica, vol. 48, no. 6, pp. 1005-1016, 2012.

[3] Z. J. Li, X. Q. Cao, and N. Ding, "Adaptive fuzzy control for synchronization of nonlinear teleoperators with stochastic time-varying communication delays," IEEE Transactions on Fuzzy Systems, vol. 19, no. 4, pp. 745-757, 2011.

[4] R. J. Anderson and M. W. Spong, "Bilateral control of teleoperators with time delay," IEEE Transactions on Automatic Control, vol. 34, no. 5, pp. 494-501, 1989.

[5] G. Niemeyer and J.-E. Slotine, "Stable adaptive teleoperation," IEEE Journal of Oceanic Engineering, vol. 16, no. 1, pp. 152-162, 1991.

[6] K. Gu, V. L. Kharitonov, and J. Chen, Stability of Time Delay Systems, Control Engineering, Birkhäuser, Basel, Switzerland, 2003.

[7] P. Arcara and C. Melchiorri, "Control schemes for teleoperation with time delay: a comparative study," Robotics and Autonomous Systems, vol. 38, no. 1, pp. 49-64, 2002.

[8] G. Niemeyer and J. E. Slotine, "Telemanipulation with time delays," International Journal of Robotics Research, vol. 23, no. 9, pp. 873-890, 2004.

[9] D. Lee and M. W. Spong, "Passive bilateral teleoperation with constant time delay," IEEE Transactions on Robotics, vol. 22, no. 2, pp. 269-281, 2006.

[10] E. Nuño, R. Ortega, N. Barabanov, and L. Basañez, "A globally stable PD controller for bilateral teleoperators," IEEE Transactions on Robotics, vol. 24, no. 3, pp. 753-758, 2008.

[11] S. Islam and P. X. Liu, "PD output feedback control design for industrial robotic manipulators," IEEE/ASME Transactions on Mechatronics, vol. 16, no. 1, pp. 187-197, 2011.

[12] S. Islam and P. X. Liu, "Robust adaptive fuzzy output feedback control system for robot manipulators," IEEE/ASME Transactions on Mechatronics, vol. 16, no. 2, pp. 288-296, 2011.
[13] R. Q. Lu, H. Y. Wu, and J. J. Bai, "New delay-dependent robust stability criteria for uncertain neutral systems with mixed delays," Journal of the Franklin Institute. Engineering and Applied Mathematics, vol. 351, no. 3, pp. 1386-1399, 2014.

[14] R. Q. Lu, H. Li, and Y. Q. Zhu, "Quantiz ed $H_{\infty}$ Filtering for Singular Time-varying Delay Systems with Unreliable Communication Channel," Circuits, Systems, and Signal Processing, vol. 31, no. 2, pp. 521-538, 2012.

[15] B. Zhou, H. Gao, Z. Lin, and G. Duan, "Stabilization of linear systems with distributed input delay and input saturation," Automatica, vol. 48, no. 5, pp. 712-724, 2012.

[16] B. Zhou, Z.-Y. Li, and Z. Lin, "Observer based output feedback control of linear systems with input and output delays," Automatica, vol. 49, no. 7, pp. 2039-2052, 2013.

[17] E. Y. Chen and W. X. Zheng, "Stability analysis of timedelay neural networks subject to stochastic perturbations," IEEE Transactions on Cybernetics, vol. 43, no. 6, pp. 2122-2134, 2013.

[18] E. Nũuo, L. Basãez, R. Ortega, and M. W. Spong, "Position tracking for non-linear teleoperators with variable time delay," International Journal of Robotics Research, vol. 28, no. 7, pp. 895910, 2009.

[19] C. C. Hua and X. P. Liu, "Delay-dependent stability criteria of teleoperation systems with asymmetric time-varying delays," IEEE Transactions on Robotics, vol. 26, no. 5, pp. 925-932, 2010.

[20] T. Namerikawa, "Bilateral control with constant feedback gains for teleoperation with time varying delay," in Proceedings of the 48th IEEE Conference on Decision and Control held jointly with 2009 28th Chinese Control Conference (CDC/CCC '09), pp. 7527-7532, December 2009.

[21] T. Imaida, Y. Yokokohji, T. Doi, M. Oda, and T. Yoshikawa, "Ground-space bilateral teleoperation experiment using ETSVII robot arm with direct kinesthetic coupling," in Proceedings of the IEEE International Conference on Robotics and Automation (ICRA '01), vol. 1, pp. 1031-1038, May 2001.

[22] M. Nohmi, M. Ando, and T. Bock, "Contact task by space teleoperation using force reflection of communication time delay," in Proceedings of the IEEE International Symposium on Computational Intelligence in Robotics and Automation, pp. 193198, June 2005.

[23] X. Wang, W. Xu, B. Liang, and C. Li, "General scheme of teleoperation for space robot," in Proceedings of the IEEE/ASME International Conference on Advanced Intelligent Mechatronics (AIM '08), pp. 341-346, Xian, China, August 2008.

[24] N. Y. Lii, Z. Chen, B. Pleintinger, C. H. Borst, G. Hirzinger, and A. Schiele, "Toward understanding the effects of visualand force-feedback on robotic hand grasping performance for space teleoperation," in Proceedings of the 23rd IEEE/RSJ 2010 International Conference on Intelligent Robots and Systems (IROS '10), pp. 3745-3752, October 2010. 


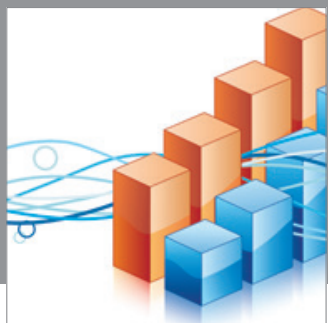

Advances in

Operations Research

mansans

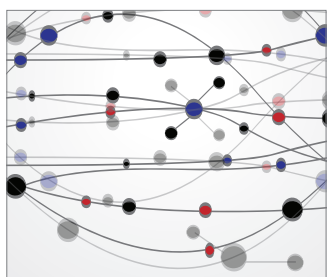

The Scientific World Journal
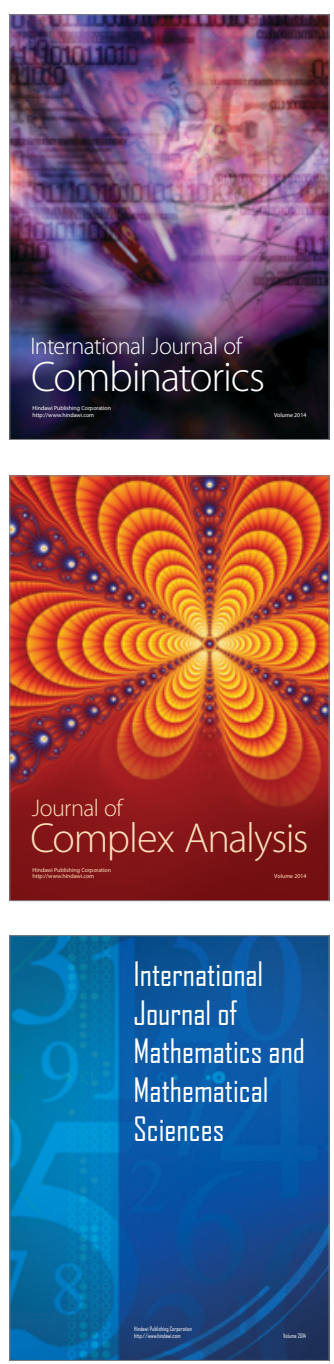
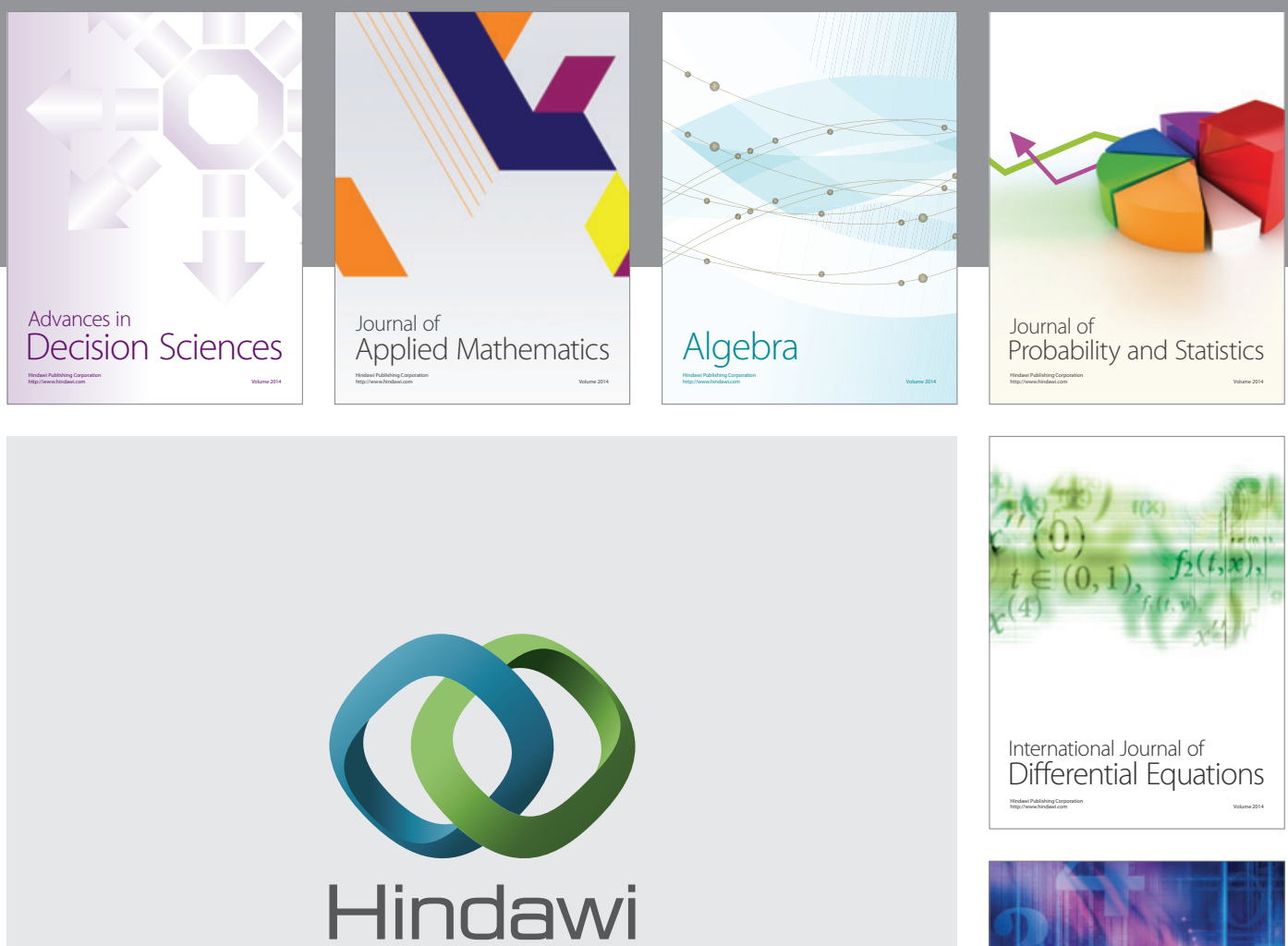

Submit your manuscripts at http://www.hindawi.com
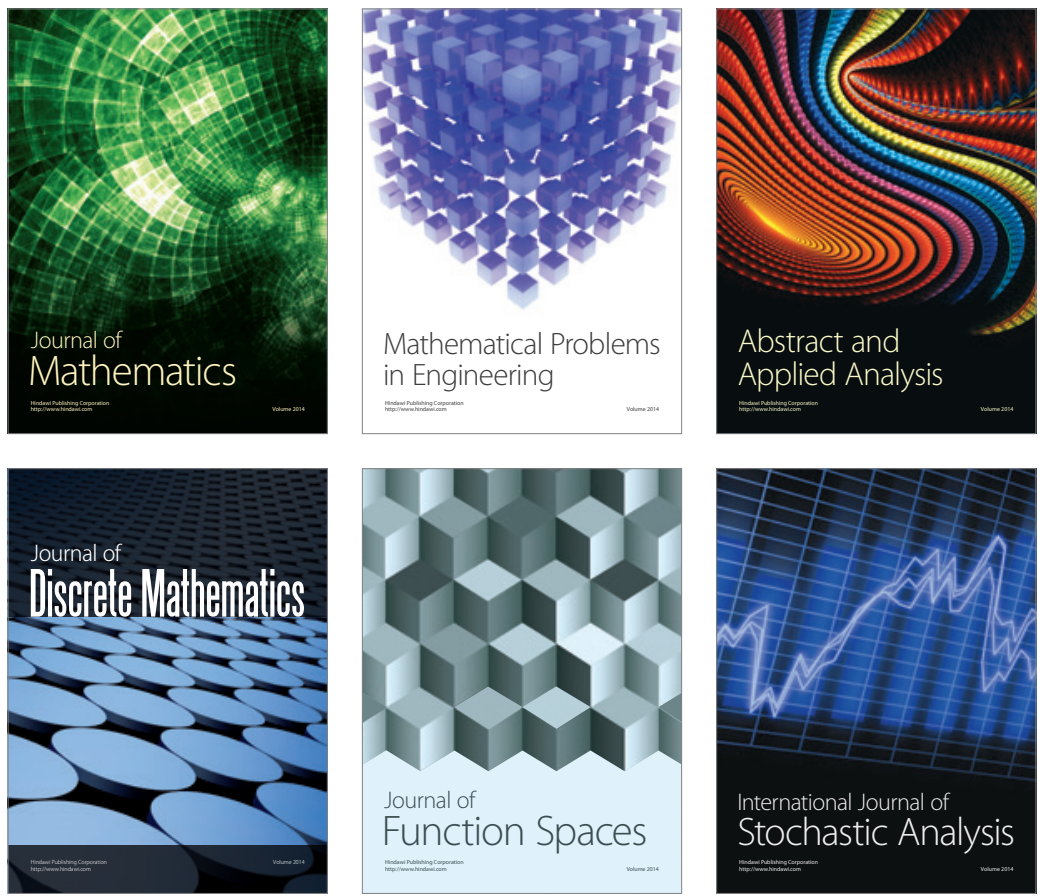

Journal of

Function Spaces

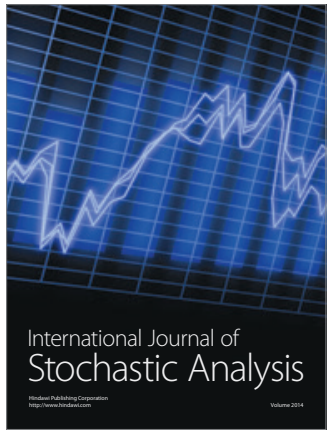

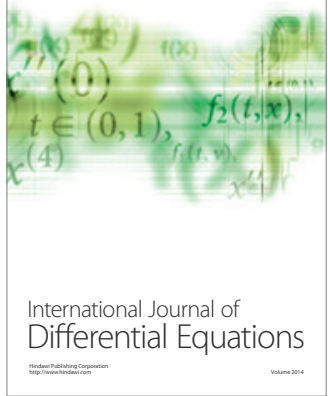
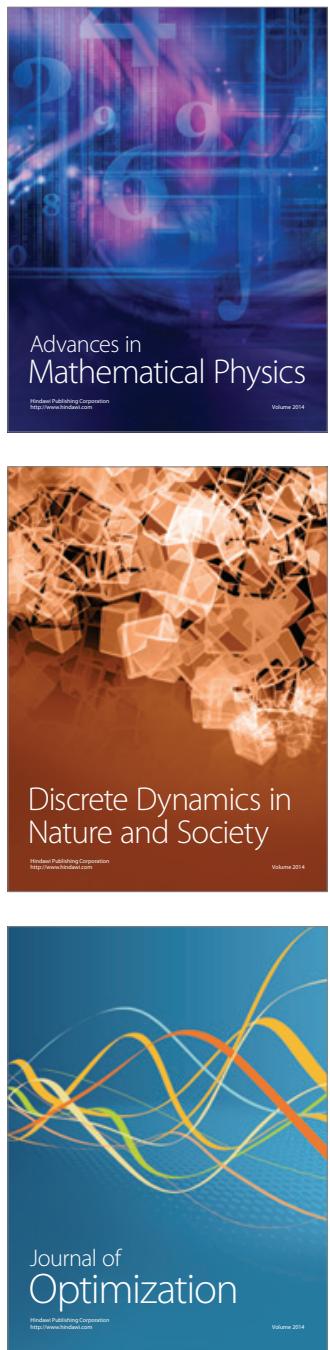